\title{
High Speed SEM Elemental Mapping with Micro-XRF-EDS
}

Andrew Menzies ${ }^{1}$, Stephan Boehm ${ }^{1}$ and John Mastovich ${ }^{2}$

${ }^{1}$ Bruker Nano GmbH, Berlin, Berlin, Germany, ${ }^{2}$ Bruker Nano Inc., Pittsburg, Pennsylvania, United States

Micro-X-ray fluorescence spectroscopy $[\mathrm{XRF}]$ is a long known and complementary analytical technique to e-beam Energy Dispersive Spectroscopy [EDS] for the characterization of the elemental composition within samples. The Bruker XTrace X-ray source enables this technique to be utilized on a Scanning Electron Microscope [SEM]. Micro-XRF X-ray excitation analysis is a supplemental technique, which also delivers small area trace analysis information. The advantages of X-ray excitation are the higher sensitivity for the detection of trace elements, higher energy X-ray line excitation, as well as information from greater depth within the sample. The availability of modern X-ray polycapillary optics enables the capability to focus X-rays to a spot size of less than $30 \mu \mathrm{m}$ all contained in an X-ray source that can be mounted on an available SEM port. X-ray energy detection uses the existing Bruker EDS detector on the SEM. Accordingly, the SEM system will subsequently have dual beam potential i.e. both an e-beam and an X-ray beam, which together offers new possibilities for material characterization.

The SEM user can operate a micro-XRF system using similar parameters as an e-beam system, yielding results comparable with traditional SEM-EDS analysis, whilst obtaining additional information from the $\mathrm{X}$-Ray-beam sample interaction. Electron excitation has a higher excitation efficiency for light elements, and it is possible to perform measurements down to Boron. X-ray excitation efficiency is better for heavy elements and allows the detection of trace concentrations down to $10 \mathrm{ppm}$ for certain elements. The larger depth of X-ray excitation allows a deeper look inside the sample, allowing the characterization of relatively thick layers or even of multilayer systems starting from $1 \mathrm{~nm}$ and ranging to $40 \mu \mathrm{m}$, which is not possible with electron excitation. Micro-XRF works with a fixed X-ray beam, consequently X-ray elemental distribution maps must be acquired via stage movement. Developments of adding a piezo-based stage (Rapid Stage) mounted on top of the SEM stage enables high-speed elemental X-ray mapping over large areas (Figure 1), thus making it suitable for the analysis of larger samples (i.e. centimeter scale) that traditionally in an SEM. Seamlessly integrated into Bruker ESPRIT software suite, it even allows for simultaneous e-beam / micro-XRF acquisition, incorporating light element spectral data as well as trace element and / or higher energy X-ray data.

Sample preparation requirements are minimal for micro-XRF analysis and thus a wide range of sample types can be analyzed (e.g no carbon coating required; no high quality polished surface required). Applications include economic geology, petrology, mining, mineral processing, forensics, environmental, metals and alloys, multilayers, and archaeology, amongst others. Figure 2 is a large area X-ray map (45 $\mathrm{mm} \times 30 \mathrm{~mm}$ ) of an Exotic copper deposit sample showing the element distribution information for copper (red), calcium (green), silicon (purple), chlorine (dark green). In addition, in this sample it is possible to detect trace elements such as Co and Sr that are not detectable by e-beam EDS as the concentrations are too low and/or the relevant element energy line is too high, and thus understand their distribution and mineralogical and textural relationships. 


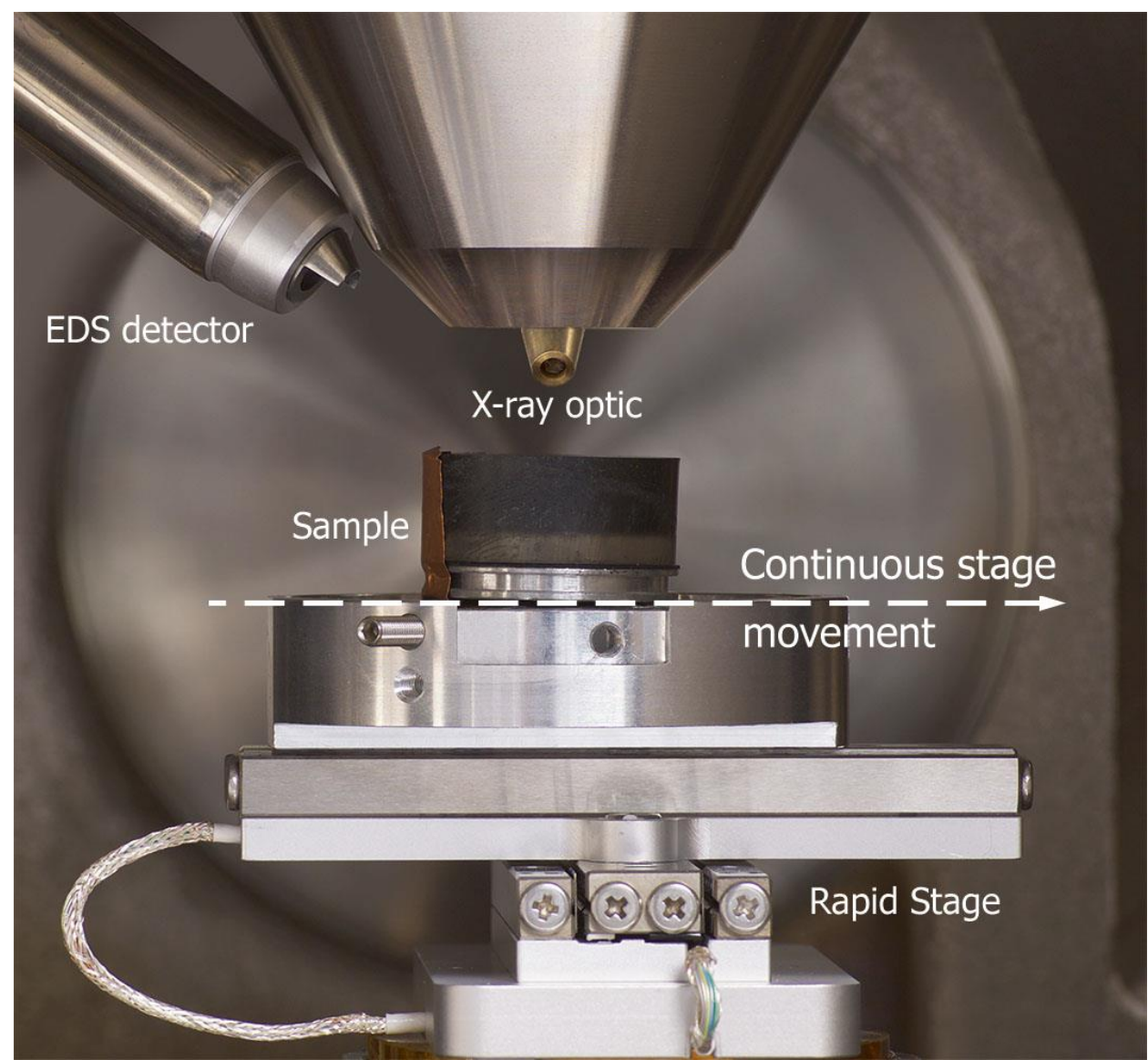

Figure 1. Example for X-ray Mapping setup in the SEM showing the Rapid Stage setup as well as the Xray optic and EDS detector placement.
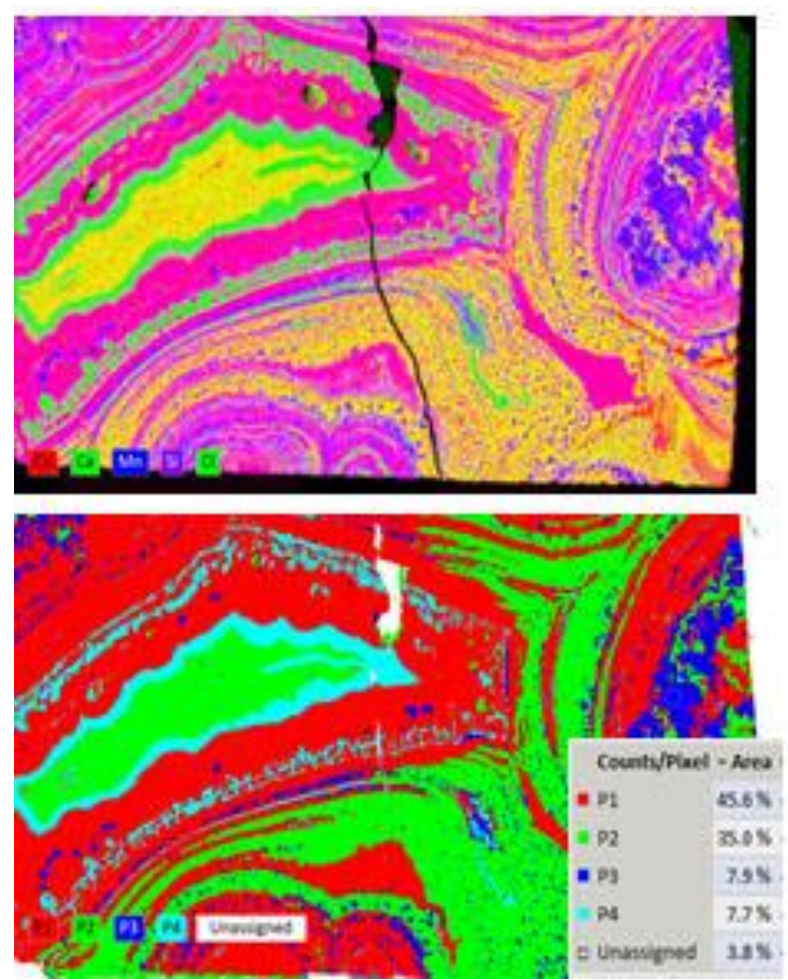
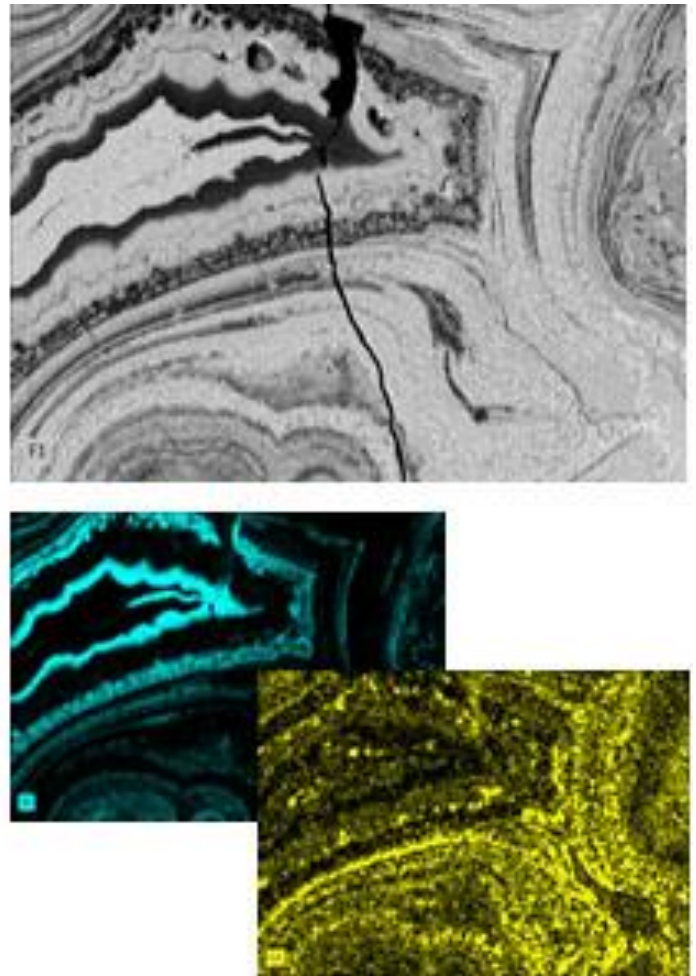
Figure 2. Large area X-ray map (45 mm x $30 \mathrm{~mm}$ ) of an Exotic cupper deposit sample aquired with XTrace (Analytical Parameters: Tube Voltage: Rh at $50 \mathrm{kV}$; Anode Current: $600 \mu \mathrm{A}$; Pixel Spacing: 25 $\mu \mathrm{m}$; Analytical Time: 101 mins). Top row ( $\mathrm{L}$ to $\mathrm{R})$ : Mixed elemental intensity map ( $\mathrm{Cu}-\mathrm{red}$; $\mathrm{Ca}-$ green; $\mathrm{Mn}$ - blue; $\mathrm{Si}$ - purple; and $\mathrm{Cl}$ - dark green; Total Xray intensity map (similar to a BSE Image); Bottom row (L to R): Phase map (P1 [Chrysocolla] - red; P2 [Atacamite] - green; P3 [Cu-Mn Wad] - blue; P4 [Carbonate] - celeste); trace element intensity maps: Sr (blue) and Co (yellow). 